\title{
Detection of aberrant crypt foci with image-enhanced endoscopy
}

\section{(ㄷ)(1) $\odot$}

Authors

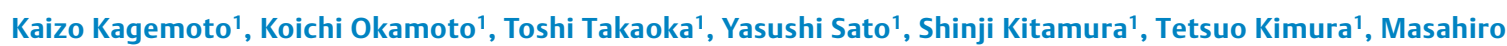
Sogabe', Hiroshi Miyamoto', Naoki Muguruma', Koichi Tsuneyama², Tetsuji Takayama'1

Institutions

1 Department of Gastroenterology and Oncology, Institute of Biomedical Sciences, Tokushima University Graduate School, Japan

2 Department of Pathology and Laboratory Medicine, Institute of Biomedical Sciences, Tokushima University Graduate School, Japan

submitted 6.1.2018

accepted after revision 11.4.2018

Bibliography

DOI https://doi.org/10.1055/a-0621-8794 |

Endoscopy International Open 2018; 06: E924-E933

(c) Georg Thieme Verlag KG Stuttgart · New York

ISSN 2364-3722

Corresponding author

Tetsuji Takayama MD, PhD, Department of

Gastroenterology and Oncology, Institute of Biomedical

Sciences, Tokushima University Graduate School, 3-18-15, Kuramoto-cho, Tokushima City, 770-8503 Japan

Fax: +81-88-633-9235

takayama@tokushima-u.ac.jp

\section{ABSTRACT}

Background and study aims Conventional detection of aberrant crypt foci (ACF) with dye-spraying and magnifying observation is labor- and skill-intensive. We performed a prospective non-inferiority study to investigate the utility of image-enhanced endoscopy (IEE) for detection of ACF.

Patients and methods Patients with a history of colorectal neoplasm were eligible. The number of ACF in the lower rectum was counted first using IEE magnification with narrow-band imaging (NBI) or blue-laser imaging (BLI), and subsequently using the methylene blue method. The primary endpoint was the ACF detection rate with IEE, i.e., the number of ACF detected with IEE relative to the number of ACF detected with methylene blue. The secondary endpoints were bowel preparation time, ACF detection time, and the detection rate with $\mathrm{NBI}$ or BLI.

Results A total of 40 patients were enrolled (NBI 20 and BLI 20). The overall detection rate for ACF with IEE was $81.7 \%$ (503/616; $95 \% \mathrm{Cl} 78.8-84.6 \%)$, meeting the primary endpoint. The detection rate for ACF with BLI $(84.9 \%, 258$ / 304) was significantly higher than with NBI (78.5\%, 245/ $312 ; P<0.05)$. Both bowel preparation time and ACF detection time were significantly shorter with IEE versus the methylene blue method ( $P<0.01$, respectively). The detection rates for dysplastic and non-dysplastic ACF with IEE were $84.4 \%(27 / 32)$ and $80.3 \%$ (469/584), respectively.

Conclusion IEE is able to detect ACF during colonoscopy with sensitivity non-inferior to that of the conventional methylene blue method. IEE is simpler than the methylene blue method and is therefore a potentially useful new tool for ACF detection.

\section{Introduction}

Aberrant crypt foci (ACF) have been described as lesions consisting of large and thick crypts densely stained with methylene blue in the colon of mice treated with a carcinogen, and they are recognized as the earliest precursor lesions of colorectal carcinogenesis [1]. ACF have also been identified on normal-appearing mucosa of surgically-resected colons from patients with colorectal cancers (CRC) with the aid of methylene blue staining under stereoscopic microscopy [2]. We previously identified human ACF in situ by spraying methylene blue under magnifying colonoscopy and showed that the number of rectal ACF in adenoma patients was significantly correlated with the number of adenomas [3]. Moreover, we and other investigators have revealed that the number of rectal ACF is significantly higher in patients with colorectal adenomas and cancers than in normal subjects. Thus, ACF are considered to be precursors to colorectal adenoma and subsequent cancer, and to be ideal 
surrogate markers for quantifying CRC risk [3-5], although some contradictory studies have also been reported [6,7]. In addition, we and other investigators recently reported randomized controlled chemoprevention trials where ACF were used as sensitive target lesions; ACF were eradicated within 2 months by treatment with candidate chemopreventive agents. The advantages of chemoprevention targeting ACF over conventional chemoprevention targeting adenomas are as follows: 1) very short-term administration of candidate agents to cancer-free subjects, resulting in; 2) infrequent occurrence of adverse effects; and 3) high compliance [8-10]. Therefore, many clinical trials focused on ACF are currently ongoing worldwide. However, the current ACF detection method with methylene blue spraying and magnified observation is very laborious and timeconsuming; mucosal staining with methylene blue including pre- and post-staining washing with water is labor-intensive and takes a long time to perform, and magnified observation close to the colorectal mucosa requires highly skilled endoscopic techniques. These aspects apparently hinder application of ACF detection in chemoprevention trials and in quantifying risk of CRC. Therefore, a new simpler ACF detection method is required.

Recently, a new image-enhanced endoscopy (IEE) method including narrow-band imaging (NBI) and blue laser imaging (BLI) has been developed. NBI in combination with magnifying endoscopy can clearly visualize the microvascular structure of lesions, which appears brownish and is a useful landmark for colorectal neoplasms, and the NBI technique has partly replaced chromoendoscopy in diagnosis of colorectal polyps $[11,12]$. By comparison, BLI comprises 2 modes of BLI and BLIbright. BLI-bright mode, because of its brightness, was reported to improve the visibility of colorectal polyps [13]. Furthermore, the combination of these IEE systems with magnifying endoscopy, which reveals precise surface and vascular patterns, was reported to be useful for predicting the histopathological diagnosis of colorectal neoplasms [14-16]. Thus, IEE combined with magnification can likely be used to observe ACF without dye-spraying. However, no published studies to date have attempted to observe ACF using IEE. In a pilot study, we were able to identify ACF using IEE (NBI/BLI) with magnification (unpublished data). Therefore, we performed the current prospective study to investigate the utility of IEE (NBI/BLI) for detection of ACF as compared with the conventional methylene blue method.

\section{Patients and methods}

\section{Patients and outcomes}

This prospective non-inferiority study was approved by the ethics committee of Tokushima University Hospital (Tokushima, Japan), and all subjects provided written informed consent. We enrolled patients with a history of colorectal neoplasm since they would be expected to have a greater number of ACF compared with normal subjects, as previously described [3,4]. Patients were required to be aged 40 to 80 years and to have a history of colorectal neoplasms or endoscopic removal of colorectal neoplasms. The exclusion criteria were as follows: (1) inflam-
Screened patients $(n=48)$

Excluded $(n=8)$

- Not meeting inclusion criteria $(n=5)$

- Refused participation $(n=3)$

- Evaluation of ACF number

$\checkmark$

IEE (randomized to $\mathrm{NBI}$ or BLI group) $(n=40)$

Methylene blue staining

1

- Detection rate (IEE / methylene blue) was analyzed

$\downarrow$

Assessment of non-inferiority of ACF detection rate with IEE (non-inferiority margin: $25 \%$ )

- Fig. 1 Flowchart of the study. IEE, image-enhanced endoscopy; ACF, aberrant crypt foci; IEE, image-enhanced endoscopy; NBI, narrow-band imaging; BLI, blue laser imaging.

matory bowel disease; (2) hereditary polyposis syndromes; (3) previous surgical resection of rectum; and (4) current use of an antithrombotic agent. The primary endpoint was the ACF detection rate with IEE, i.e., the number of ACF detected with IEE (NBI/BLI) relative to the number of ACF detected with methylene blue staining. Secondary endpoints compared the following parameters between the IEE and methylene blue methods: (1) bowel preparation time for ACF observation, including washing and staining time; (2) volume of water used for washing; and (3) ACF observation time under magnifying endoscopy. Additional analyses were performed to identify dysplastic and non-dysplastic ACF endoscopically using IEE (NBI/BLI) by assessing the validity of the endoscopic diagnosis based on the degree of agreement among IEE, methylene blue, and the histological diagnosis, and detection rates for both types of ACF with IEE were evaluated. Fig. 1 shows the flowchart for this study. This trial was registered in the University Hospital Medical Information Network Clinical Trials Registry (UMIN000022322).

\section{Endoscopic equipment}

NBI procedures were performed with PCF-Q260ZI endoscopes in combination with an EVIS LUCERA processor (Olympus Corp., Tokyo, Japan) $[17,18]$. BLI procedures were performed with EC-L590ZW endoscopes in combination with a LASEREO laser endoscope system (Fujifilm Corp., Tokyo, Japan) [15, 19].

\section{Definition of ACF with methylene blue method}

We defined a tiny lesion that consists of larger crypts, thicker epithelial lining, and staining darker than normal epithelial crypts in the methylene blue-stained regions of the colorectum as ACF, according to previous studies $[3,10]$. Any lesion that 
was apparently protruded from the surrounding normal mucosa under a white light observation was categorized into polyps such as hyperplastic and/or adenomatous polyps and excluded.

\section{Definition of ACF with IEE}

We first performed a pilot study to define the endoscopic appearance of ACF with IEE. We were able to find a small lesion that consists of large crypts with white pericryptal zones and shows a distinct margin in the lower rectal region by using magnified NBI observation ( Supplementary Fig. 1a). This finding was similar to that of a magnified image of ACF with methylene blue staining, as previously described $[3,10]$. To verify the diagnosis, the adjacent mucosa was marked with argon plasma coagulation (APC) and then methylene blue staining was performed as a gold standard on the referral area for ACF observation. We were able to identify typical ACF, which consisted of large crypts densely stained with methylene blue in the corresponding location ( Supplementary Fig. 1b). We were also able to identify ACF by BLI magnification and the findings were similar to those with NBI. We observed a total of 64 ACFs in six patients with adenomas using IEE and verified that all of these were ACF by methylene blue staining. Thus, we defined a tiny lesion that consists of large crypts with white pericryptal zones and shows a distinct margin, identified by IEE magnification, as ACF.

\section{Procedure}

Bowel preparation for colonoscopy was carried out using polyethylene glycol solution (Niflec: Ajinomoto Corp., Tokyo, Japan). When the endoscope was pulled back to the rectum after total colonoscopy, the rectum from the middle Houston valve to the dentate line was washed thoroughly with water to remove sticky mucus, and then ACF were observed in the lower rectal region using IEE. With regard to IEE observation, patients were randomly assigned to either the $\mathrm{NBI}$ or BLI group using a sealed-envelope method. For IEE (NBI/BLI) observation, we detected the tiny lesion with low magnification at a far field of view and subsequently zoomed in on the lesion to observe its precise appearance under high magnification. In the BLI group, BLI-bright mode was used mainly to screen ACF with low magnification at a far field, and BLI mode was used for magnified observation at a near field. After IEE observation, we sprayed methylene blue onto the lower rectum, and waited for more than 2 minutes until the mucosa was stained sufficiently. The mucosal surface was then thoroughly washed again with water. When small regions densely stained with methylene blue at low magnification were found, we zoomed in on them and observed the precise appearance under high magnification with white light imaging (WLI). ACF specimens were obtained by endoscopic biopsy as described previously [20]. A histopathological diagnosis of ACF, including dysplastic and non-dysplastic types, was made by one pathologist (K.T), according to previous reports $[3,21]$. ACF detection with IEE and the methylene blue method were both performed by the same endoscopist. Colonoscopies were performed with patients under conscious sedation with midazolam. All colonoscopies were performed by three endoscopists who had each performed more than
1000 colonoscopies. They also had experience with more than 20 cases of ACF detection using IEE and methylene blue before the start of this study.

\section{Sample size and statistics}

This was a single-institution prospective study to verify the hypothesis that IEE is able to detect ACF with non-inferior sensitivity to that of the methylene blue method as the gold standard. In our pilot study, a total of 64 ACF were detected with IEE $(\mathrm{NBI}=33, \mathrm{BLI}=31)$ and $81 \mathrm{ACF}$ with the methylene blue method, indicating that the detection rate with IEE was $79.0 \%$ $(\mathrm{NBI}=78.6 \%, \mathrm{BLI}=79.5 \%)$. We considered that it would be acceptable if an ACF detection rate with IEE of at least $75 \%$ was achieved. Thus, we set a one-sided non-inferiority margin of $25 \%$. With a power of $80 \%$ and a one-sided significance level of $5 \%$, a total of 246 ACF were required. In our previous study, the mean number of ACF in patients with adenoma was seven, therefore, the sample size needed was calculated to be 40 patients. The detection rates between the two groups (NBI/BLI) were compared using Student's $t$ test. Washing and staining time, volume of water used, and observation time were also compared between the IEE and methylene blue groups using Student's $t$ test, and $P<0.05$ was considered to be statistically significant.

\section{Results}

\section{Patients}

A total of 40 eligible patients were randomly assigned to the $\mathrm{NBI}$ and BLI groups (20 patients per group) ( $\triangleright$ Fig. 1 ). The clinical characteristics of the patients in the two groups were well balanced ( $\triangleright$ Table 1$)$.

\section{Detection rate for ACF with IEE}

We evaluated the rate of detection of ACF with IEE using the number of ACF detected with the methylene blue method as a reference. A total of 503 ACFs were detected with IEE (NBI/BLI) and 616 ACFs were detected with the methylene blue method in this cohort. Therefore, the overall detection rate with IEE was $81.7 \%(503 / 616 ; 95 \% \mathrm{Cl} 78.8-84.6 \%)$, indicating that the primary endpoint was achieved ( $>$ Table 2 ). In the NBI group, 245 ACFs were detected with $\mathrm{NBI}$ and 312 ACFs were detected with methylene blue; the detection rate for ACF with NBI was $78.5 \%$ (245/312; 95\%Cl 75.3-81.7\%). In the BLI group, 258 ACFs were detected with BLI and 304 ACFs were detected with methylene blue; the detection rate for ACF with BLI was $84.9 \%$ (258/304; $95 \% \mathrm{Cl} 80.4-89.4 \%)$. The ACF detection rate with BLI was significantly higher than that with NBI $(P<0.05)(\triangleright$ Table2).

\section{Washing and staining time, washing water volume, and ACF observation time}

To evaluate labor- and time-intensity for ACF observation with IEE, we compared bowel preparation time for ACF observation, volume of water used, and ACF observation time between IEE and the methylene blue method in 40 cases ( $>$ Table 3 ). Mean bowel preparation time, including washing and staining time, was significantly shorter with IEE versus the methylene blue 
Table 1 Patient characteristics.

\begin{tabular}{|l|l|l|l|}
\hline & IEE $(\mathbf{n = 4 0 )}$ & $\mathbf{N B I}(\mathbf{n = 2 0 )}$ & BLI (n=20) \\
\hline Male/Female & $20 / 20$ & $10 / 10$ & $10 / 10$ \\
\hline Age (y, mean \pm SD) & $67.0 \pm 8.7$ & $68.8 \pm 6.1$ & $65.4 \pm 10.3$ \\
\hline History of colorectal tumor & 40 & 20 & 20 \\
\hline " Cancer & 3 & 1 & 2 \\
\hline - Adenoma & 40 & 20 & 20 \\
\hline IEE, image-enhanced endoscopy; NBI, narrow-band imaging; BLI, blue laser imaging; SD, standard deviation.
\end{tabular}

$\checkmark$ Table 2 Detection rate for ACF with IEE.

\begin{tabular}{|l|l|l|l|}
\hline & Number of ACF detected with IEE & Number of ACF detected with methylene blue & Detection rate (95\%CI) \\
\hline IEE & 503 & 616 & $81.7(78.8-84.6)$ \\
\hline $\mathrm{NBI}$ & 245 & 312 & $78.5(75.3-81.7)$ \\
\hline $\mathrm{BLI}$ & 258 & 304 & $84.9(80.4-89.4)$ \\
\hline
\end{tabular}

Detection rate was expressed as percentage (\%). ACF, aberrant crypt foci;[]|EE, image-enhanced endoscopy; NBI, narrow-band imaging; BLI, blue laser imaging; CI, confidence interval.

- Table 3 Evaluation of convenience for ACF observation.

\begin{tabular}{|c|c|c|c|}
\hline & IEE $(n=40)$ & Methylene blue $(n=40)$ & $P$ value \\
\hline Bowel preparation time including washing and staining time (min, mean \pm SD) & $1.1 \pm 0.4$ & $8.2 \pm 2.3$ & $<0.01$ \\
\hline Volume of water used ( $\mathrm{mL}$, mean $\pm \mathrm{SD}$ ) & $85.0 \pm 30.3$ & $452.5 \pm 187.6$ & $<0.01$ \\
\hline ACF observation time (min, mean \pm SD) & $5.2 \pm 1.9$ & $7.2 \pm 2.7$ & $<0.01$ \\
\hline
\end{tabular}

method $(1.1 \pm 0.40$ vs $8.2 \pm 2.3 \mathrm{~min} ; P<0.01)$. Mean volume of water used was significantly lower with IEE than with the methylene blue method $(85.0 \pm 30.3$ vs $452.5 \pm 187.6 \mathrm{~mL} ; P<$ 0.01). Moreover, mean ACF observation time was significantly shorter with IEE versus the methylene blue method $(5.2 \pm 1.9$ vs $7.2 \pm 2.7 \mathrm{~min} ; P<0.01)$.

\section{Endoscopic appearance and histological validation of non-dysplastic and dysplastic ACF with IEE}

Because two distinct endoscopic features of dysplastic and nondysplastic ACF with the methylene blue method have been reported $[3,21]$, we investigated the morphological features of dysplastic and non-dysplastic ACF detected with IEE as compared with methylene blue staining and histological findings. We found two morphological types of ACF using IEE with magnification. Representative endoscopic findings of these ACFs using $\mathrm{NBI}$ and methylene blue are shown in > Fig. 2. One is a focus that consists of large crypts with regularly arranged white pericryptal zones and shows a distinct margin ( $>$ Fig. 2a). Methylene blue staining of the focus revealed large crypts with a thicker epithelial lining and a larger pericryptal zone than normal crypts ( $\mathbf{F i g . 2 b}$ ), consistent with previous reports $[3,10]$. Histologically, there was enlargement and elongation of the ducts, but not dysplasia ( $\mathbf{F i g . 2 c}$ ). Thus, this type of ACF was non-dysplastic ACF. The other type of ACF consisted of large crypts with a white pericryptal zone, but the border of each crypt (pericryptal zone) was indistinct or invisible and showed a distinct margin ( $>$ Fig. $\mathbf{2 d}$ ). Methylene blue staining revealed that the epithelial linings were thicker than those of non-dysplastic ACF, and the crypt lumens were compressed or unclear ( Fig.2e). Histopathological examination demonstrated hyperchromatic nuclei with stratification and loss of polarity, indicating that this type of ACF is dysplastic ACF ( $\triangleright$ Fig. 2f). Features of non-dysplastic and dysplastic ACF detected with BLI were similar to those with NBI ( $\vee$ Fig. 3 ).

We then assessed the validity of endoscopic diagnosis by examining the degree of agreement between the IEE and methylene blue diagnosis, and histological findings in 72 randomly obtained samples from 26 patients. Diagnosis with IEE and methylene blue, and histology, was concordant in 61 specimens without dysplasia $(\mathrm{NBI}=30, \mathrm{BLI}=31)$ and 9 specimens with dysplasia $(\mathrm{NBI}=3, \mathrm{BLI}=6)$. There was discordance in two specimens; one focus was identified as dysplastic by NBI but it was diagnosed as non-dysplastic by methylene blue and histological examination. The other focus was identified as non-dysplastic 

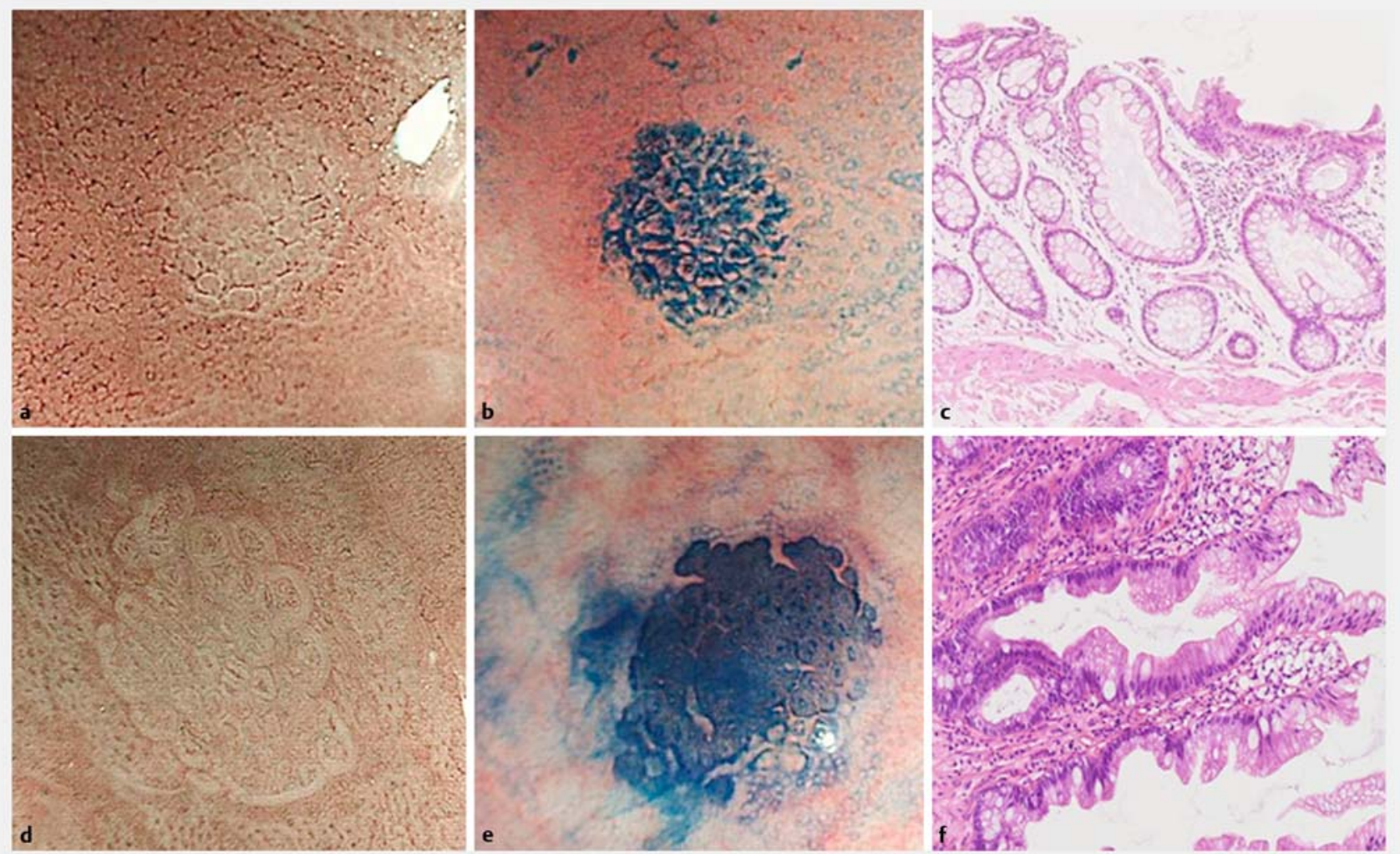

- Fig. 2 Endoscopic findings of aberrant crypt foci (ACF) using narrow-band imaging (NBI) and methylene blue, and histological findings. a-c Representative images of non-dysplastic ACF. a One focus that consists of large crypts with a regularly arranged white pericryptal zone under NBI magnification. b Methylene blue staining of this focus revealed that the large crypts had a thicker epithelial lining and a larger pericryptal zone than normal crypts. c Histologically, there was enlargement and elongation of the ducts, but not dysplasia (orig. mag. $\times 10$ ). $\mathbf{d - f}$ Representative images of dysplastic ACF. $\mathbf{d}$ One focus consisted of large crypts with a white pericryptal zone, but the border of each crypt was indistinct or invisible. e Methylene blue staining revealed that the epithelial lining was thicker than in non-dysplastic ACF, and the crypt lumen was compressed or unclear. $\mathbf{f}$ Histologically, there were hyperchromatic nuclei with stratification, and loss of polarity (orig. mag. $\times 10$ ).

by BLI and methylene blue; however, it was diagnosed as normal epithelia by histological examination ( $\bullet$ Table 4$)$.

Based on these results, we evaluated the diagnostic yield for IEE and methylene blue in non-dysplastic and dysplastic ACF in this cohort. In the dysplastic group, both IEE and methylene blue were found to have high values $(90 \%-100 \%)$ for sensitivity, specificity, positive and negative predictions, and accuracy, with the exception of the positive predictive value of NBI (75\%)

\section{$(\triangleright$ Table 5).}

\section{Detection rate for non-dysplastic and dysplastic ACF with IEE}

Because good detectability of non-dysplastic and dysplastic ACF with IEE was demonstrated, we next evaluated the detection rate for non-dysplastic and dysplastic ACF with IEE compared with methylene blue staining as a reference. ACFs from all 40 patients in this study were evaluated, i.e., 32 dysplastic and 584 non-dysplastic ACFs diagnosed based on methylene blue staining ( $\sim$ Table 6 ). Of these, 27 dysplastic ACF could be detected by IEE. Therefore, the detection rate for dysplastic ACF was $84.4 \%(27 / 32 ; 95 \% \mathrm{Cl} 75.2-93.5 \%) ; \mathrm{NBI}=81.3 \%(13 / 16)$ and $B L I=87.5 \%(14 / 16)$. There was no significant difference be- tween NBI and BLI. Similarly, the detection rate for non-dysplastic ACF with IEE was $80.3 \%(469 / 584 ; 95 \% \mathrm{Cl} 76.8-83.8 \%)$; NBI $=77.0 \%(228 / 296)$ and $\mathrm{BLI}=83.7 \%(241 / 288)$, and there was a significant difference between NBI and BLI $(P<0.05)$.

\section{Discussion}

This is the first trial to evaluate the utility of IEE (NBI/BLI) on ACF detection during colonoscopy. In this trial, we showed that the detection rate for ACF with IEE was $81.7 \%$ (95\%CI 78.8-84.6\%), indicating that the IEE method is non-inferior to conventional methylene blue staining for detection of ACF, and thus the primary endpoint was achieved. Moreover, we demonstrated that bowel preparation time, volume of water used, and ACF observation time with IEE were significantly shorter than with methylene blue staining, suggesting that IEE is much easier and simpler to perform than the conventional methylene blue method. Furthermore, our results suggest that IEE is able to discriminate dysplastic ACF from non-dysplastic ACF. Thus, we demonstrated the utility of IEE in detecting ACF without dye-spraying for quantification of CRC risk and/or evaluation of the effects of chemopreventive agents. Although further studies are required 

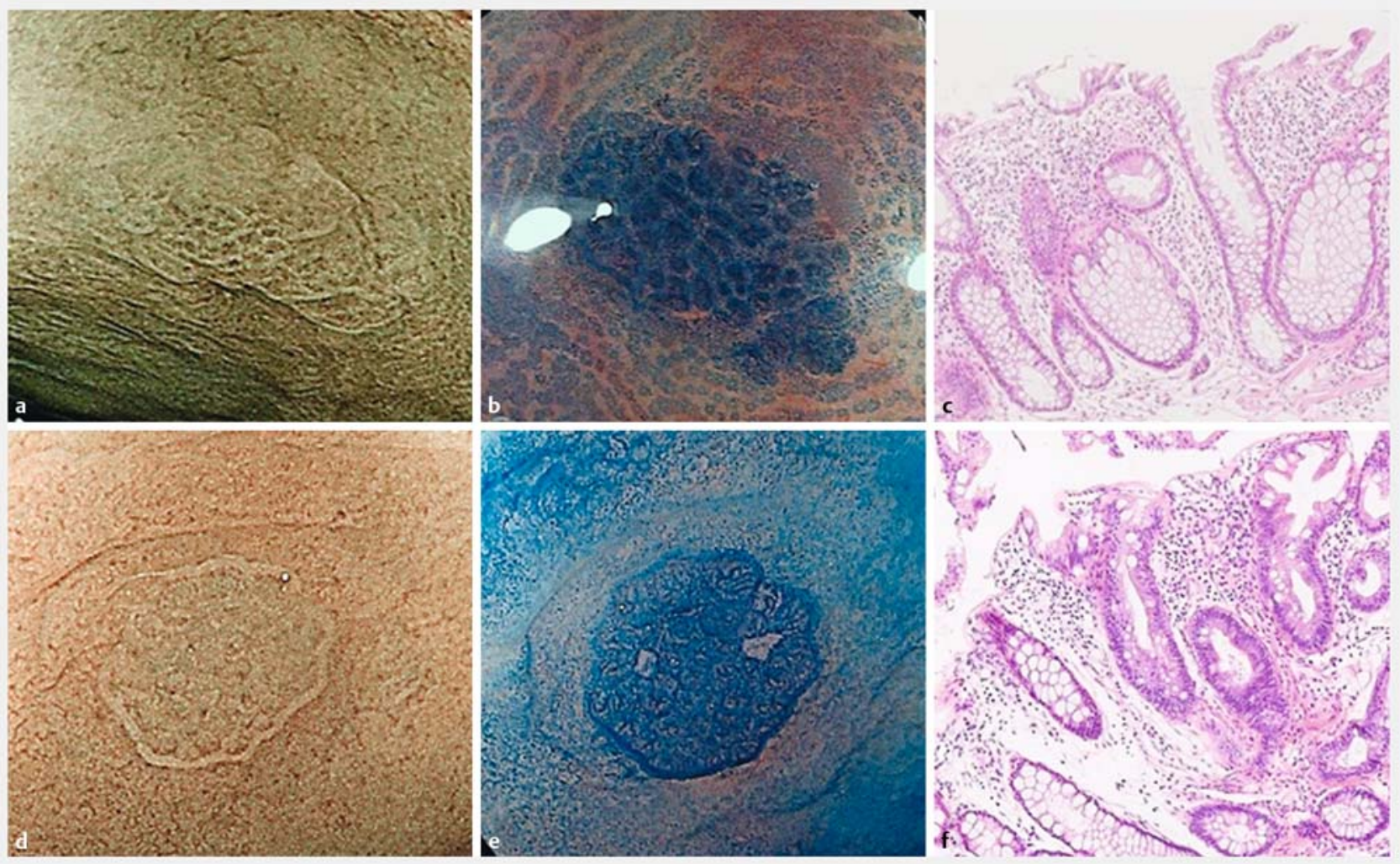

- Fig. 3 Endoscopic findings of ACF with blue laser imaging (BLI) and methylene blue staining, and histological findings. Features of non-dysplastic and dysplastic ACF detected with BLI were similar to those with narrow-band imaging. a-c Representative images of non-dysplastic ACF. d-f Representative images of dysplastic ACF.

- Table 4 Histological validation of ACF diagnosis with IEE and methylene blue.

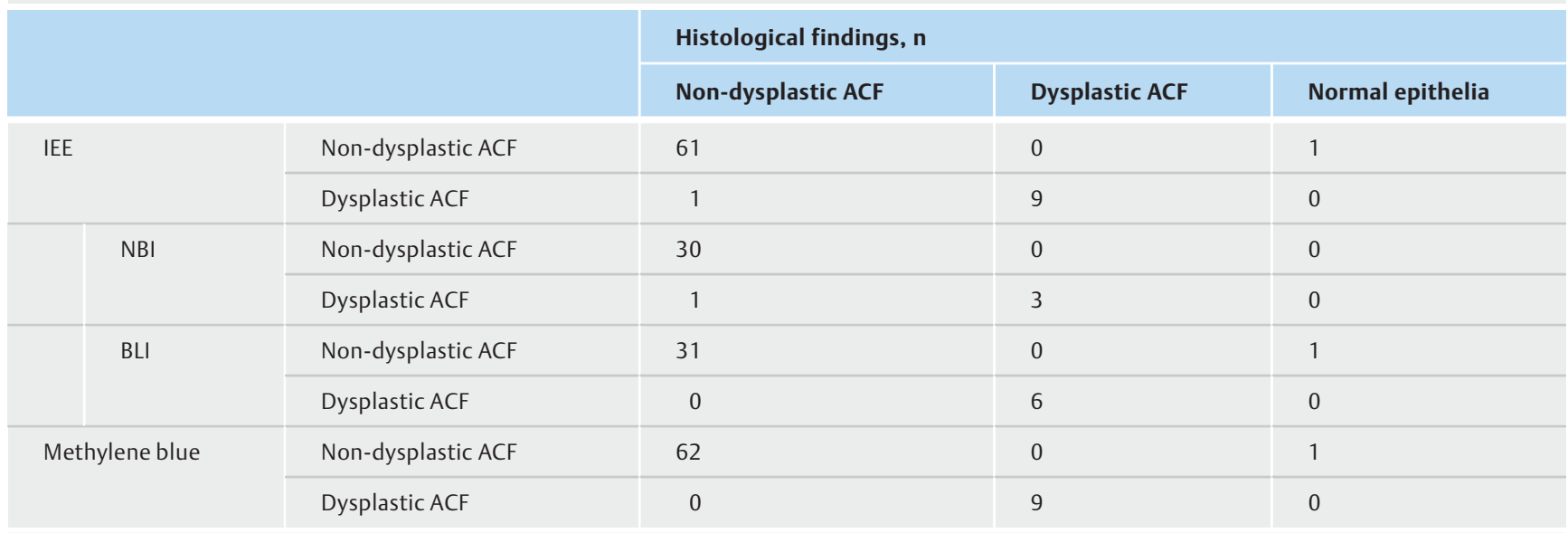

ACF, aberrant crypt foci; IEE, image-enhanced endoscopy; NBI, narrow-band imaging; BLI, blue laser imaging.

to validate our findings, IEE may replace the conventional methylene blue method for ACF detection.

A subgroup analysis of this study revealed that the detection rate for ACF with BLI (84.9\%) was significantly higher than with $\mathrm{NBI}(78.5 \%$; $P<0.05)$. This may be partially explained by the fact that BLI-bright has a longer observable distance and higher contrast even in the far field of view as compared with NBI mode [19]. This is important for ACF observation because endoscopists typically attempt to identify ACF on the colorectal mucosa at low magnification as the initial step. If a tiny ACF-suspicious lesion is found, it is then observed at higher magnification to reach an accurate diagnosis of ACF. A recent randomized controlled study revealed that NBI did not improve detection of colorectal polyps compared with WLI [18]. Conversely, a recent 
Table 5 Identification of dysplastic ACF with IEE and methylene blue.

\begin{tabular}{|c|c|c|c|c|c|c|c|}
\hline & & & Sensitivity & Specificity & $\begin{array}{l}\text { Positive } \\
\text { predictive value }\end{array}$ & $\begin{array}{l}\text { Negative } \\
\text { predictive value }\end{array}$ & Diagnostic accuracy \\
\hline \multirow[t]{4}{*}{ Dysplastic ACF } & \multicolumn{2}{|l|}{ IEE } & 100 & 98.4 & 90 & 100 & 98.6 \\
\hline & & $\mathrm{NBI}$ & 100 & 96.8 & 75 & 100 & 97.1 \\
\hline & & BLI & 100 & 100 & 100 & 100 & 100 \\
\hline & \multicolumn{2}{|c|}{ Methylene blue } & 100 & 100 & 100 & 100 & 100 \\
\hline
\end{tabular}

All values are expressed as percentages. ACF, aberrant crypt foci; IEE, image-enhanced endoscopy; NBI, narrow-band imaging; BLI, blue laser imaging.

- Table 6 Endoscopic diagnosis of ACF with IEE and methylene blue method.

\begin{tabular}{|c|c|c|c|}
\hline & & \multicolumn{2}{|l|}{ Methylene blue, n } \\
\hline & & Non-dysplastic ACF & Dysplastic ACF \\
\hline \multirow[t]{3}{*}{$\operatorname{IEE}(n=40)$} & Non-dysplastic ACF & 469 & 1 \\
\hline & Dysplastic ACF & 6 & 27 \\
\hline & Undetected & 109 & 4 \\
\hline \multirow[t]{3}{*}{$\mathrm{NBI}(\mathrm{n}=20)$} & Non-dysplastic ACF & 228 & 1 \\
\hline & Dysplastic ACF & 3 & 13 \\
\hline & Undetected & 65 & 2 \\
\hline \multirow[t]{3}{*}{$\mathrm{BLI}(\mathrm{n}=20)$} & Non-dysplastic ACF & 241 & 0 \\
\hline & Dysplastic ACF & 3 & 14 \\
\hline & Undetected & 44 & 2 \\
\hline
\end{tabular}

randomized controlled study showed that BLI-bright mode significantly improved detectability of colorectal polyps compared with WLI [22]. These results are consistent with our results showing that BLI-bright was superior to NBI for detecting ACF.

In this study, bowel preparation time, including washing and staining time, volume of water used and ACF observation time with IEE were significantly shorter than with the methylene blue method. These results are quite reasonable because IEE does not require dye-spraying, washing the mucosa to remove sticky mucus and excess dye, etc. However, the shorter ACF observation time with IEE might have been partly due to the lower number of ACF detected as compared with methylene blue.

We also showed that IEE was able to discriminate between dysplastic ACF and non-dysplastic ACF (NBI and BLI detection rate of dysplastic ACF, $81.3 \%$ and $87.5 \%$, respectively) without methylene blue staining ( $\bullet$ Table 6 ). This is particularly important because dysplastic ACF are known to be strongly associated with colorectal adenomas and subsequent cancers. IEE is an endoscopic technology that emphasizes mucosal microstructure (surface pattern) and microvessels (vascular pattern) [23-26]. NBI and BLI mode visualized non-dysplastic ACF as foci that consisted of dilated crypts with regularly arranged white pericryptal zones. In contrast, dysplastic ACF were visualized as foci that consisted of dilated crypts with white pericryp- tal zones, but each pericryptal zone (the border of each crypt) was indistinct or invisible ( $\mathbf{F i g . 2 , ~}$ - Fig.3). Notably, these differences in endoscopic images depend on differences in the surface pattern but not on the vascular pattern (neovascularization). Histopathological examination of these ACF also revealed that the differences depend on cellular and structural atypia but not on the density of microvessels. These results suggest that proliferation of microvessels within ACF (both dysplastic and non-dysplastic ACF) may be lower than within adenomas, probably due to the fact that ACF are very small lesions $(<1$ to $2 \mathrm{~mm})$. Therefore, the surface pattern in IEE (NBI or BLI) with magnification would be an essential factor for discrimination between dysplastic ACF and non-dysplastic ACF.

There are some limitations in our study. First, it was performed at a single institute with a small number of cases, and therefore there may have been some degree of selection bias. Second, both IEE and methylene blue method were performed by the same endoscopist, and thus could have been subject to observer bias. In addition, we evaluated the detection rate for ACF with IEE including NBI and BLI together. However, because a significant difference in detection rate was observed between the NBI and BLI groups in a subgroup analysis, a larger-scale randomized controlled trial that investigates non-inferiority of 
$\mathrm{NBI}$ or BLI independently to the dye-spraying method should be performed.

NBI was performed using NBI (PCF-Q260ZI) with an EVIS LUCERA system, which was generally used in Japan at the start of this study. Recently, a new-generation brighter NBI system, LUCERA Elite or Eves Exera III (Olympus Corp.), has been developed $[27,28]$. More recently, it has been reported that the next-generation NBI significantly improved detection of colorectal neoplasms in comparison with WLI. Because the main benefit of these NBI systems is brightness [29], IEE with enhanced brightness would potentially be useful for detecting ACF as well as adenomas. Furthermore, a new IEE modality, Linked Color Imaging ( $\mathrm{LCl}$ ), developed by Fujifilm Corporation (Tokyo, Japan) is also expected to improve detection and discrimination of gastrointestinal neoplasia [30,31]. Therefore, these systems might be useful for detection and discrimination of ACF. Other IEE systems such as flexible spectral imaging color enhancement (FICE) (Fujifilm Corp.) and i-SCAN (Pentax Corp.) may also be useful for ACF detection. However, because these modalities did not reportedly improve adenoma detection rates [32,33], this possibility seems unlikely. Further study will be required to identify "optimal” IEEs for ACF detection.

\section{Conclusion}

In conclusion, our study showed that IEE (NBI/BLI) with magnified observation is useful for detection of ACF. Moreover, preparation time and observation time for ACF detection with IEE were much shorter than with conventional methylene blue staining and the difference between methods was statistically significant, indicating that IEE is much easier and simpler to perform than methylene blue staining for detection of ACF. Among the IEE modalities, BLI showed a significantly higher detection rate for ACF as compared with NBI. In addition, our results indicate that these IEE modalities may be able to discriminate dysplastic ACF from non-dysplastic ACF.

\section{Competing interests}

\section{None}

\section{References}

[1] Bird RP. Observation and quantification of aberrant crypts in the murine colon treated with a colon carcinogen: preliminary findings. Cancer Lett 1987; 37: 147-151

[2] Pretlow TP, Brasitus TA, Fulton NC et al. K-ras mutations in putative preneoplastic lesions in human colon. J Natl Cancer Inst 1993; 85: 2004-2007

[3] Takayama T, Katsuki S, Takahashi Y et al. Aberrant crypt foci of the colon as precursors of adenoma and cancer. N Engl J Med 1998; 339 : $1277-1284$

[4] Kim J, Ng J, Arozulllah A et al. Aberrant crypt focus size predicts distal polyp histopathology. Cancer Epidemiol Biomarkers Prev 2008; 17: $1155-1162$
[5] Seike K, Koda K, Oda K et al. Assessment of rectal aberrant crypt foci by standard chromoscopy and its predictive value for colonic advanced neoplasms. Am J Gastroenterol 2006; 101: 1362 - 1369

[6] Cho NL, Redston M, Zauber AG et al. Aberrant crypt foci in the adenoma prevention with celecoxib trial. Cancer Prev Res (Phila) 2008; 1 : $21-31$

[7] Mutch MG, Schoen RE, Fleshman JW et al. A multicenter study of prevalence and risk factors for aberrant crypt foci. Clin Gastroenterol Hepatol 2009; 7: 568-574

[8] Shpitz B, Klein E, Buklan G et al. Suppressive effect of aspirin on aberrant crypt foci in patients with colorectal cancer. Gut 2003; 52: $1598-1601$

[9] Takayama T, Nagashima H, Maeda M et al. Randomized double-blind trial of sulindac and etodolac to eradicate aberrant crypt foci and to prevent sporadic colorectal polyps. Clin Cancer Res 2011; 17: 3803 3811

[10] Hosono K, Endo H, Takahashi H et al. Metformin suppresses colorectal aberrant crypt foci in a short-term clinical trial. Cancer Prev Res (Phila) 2010; 3: $1077-1083$

[11] Inoue T, Murano M, Murano $\mathrm{N}$ et al. Comparative study of conventional colonoscopy and pan-colonic narrow-band imaging system in the detection of neoplastic colonic polyps: a randomized, controlled trial. J Gastroenterol 2008; 43: 45-50

[12] Adler A, Aschenbeck J, Yenerim T et al. Narrow-band versus whitelight high definition television endoscopic imaging for screening colonoscopy: a prospective randomized trial. Gastroenterology 2009; 136: $410-416$

[13] Yoshida N, Hisabe T, Hirose R et al. Improvement in the visibility of colorectal polyps by using blue laser imaging (with video). Gastrointest Endosc 2015; 82: 542 - 549

[14] Wada Y, Kudo SE, Kashida $\mathrm{H}$ et al. Diagnosis of colorectal lesions with the magnifying narrow-band imaging system. Gastrointest Endosc 2009; 70: 522-531

[15] Yoshida N, Yagi N, Inada Y et al. Ability of a novel blue laser imaging system for the diagnosis of colorectal polyps. Dig Endosc 2014; 26: $250-258$

[16] Yoshida N, Hisabe T, Inada Y et al. The ability of a novel blue laser imaging system for the diagnosis of invasion depth of colorectal neoplasms. J Gastroenterol 2014; 49: $73-80$

[17] Katagiri A, Fu KI, Sano Y et al. Narrow band imaging with magnifying colonoscopy as diagnostic tool for predicting histology of early colorectal neoplasia. Aliment Pharmacol Ther 2008; 27: 1269-1274

[18] Ikematsu H, Saito Y, Tanaka S et al. The impact of narrow band imaging for colon polyp detection: a multicenter randomized controlled trial by tandem colonoscopy. J Gastroenterol 2012; 47: 1099-1107

[19] Kaneko K, Oono Y, Yano T et al. Effect of novel bright image enhanced endoscopy using blue laser imaging (BLI). Endosc Int Open 2014; 2: E212-219

[20] Takayama T, Ohi M, Hayashi T et al. Analysis of K-ras, APC, and betacatenin in aberrant crypt foci in sporadic adenoma, cancer, and familial adenomatous polyposis. Gastroenterology 2001; 121: $599-$ 611

[21] Takayama T, Miyanishi K, Hayashi T et al. Aberrant crypt foci: detection, gene abnormalities, and clinical usefulness. Clin Gastroenterol Hepatol 2005; 3: S42-45

[22] Ikematsu H, Sakamoto T, Togashi K et al. Detectability of colorectal neoplastic lesions using a novel endoscopic system with blue laser imaging: a multicenter randomized controlled trial. Gastrointest Endosc 2017; 86: 386-394

[23] Muto M, Minashi K, Yano T et al. Early detection of superficial squamous cell carcinoma in the head and neck region and esophagus by narrow band imaging: a multicenter randomized controlled trial. J Clin Oncol 2010; 28: 1566-1572 
[24] Ezoe $\mathrm{Y}$, Muto $\mathrm{M}$, Uedo $\mathrm{N}$ et al. Magnifying narrowband imaging is more accurate than conventional white-light imaging in diagnosis of gastric mucosal cancer. Gastroenterology 2011; 141: 2017-2025

[25] Hirata M, Tanaka S, Oka S et al. Evaluation of microvessels in colorectal tumors by narrow band imaging magnification. Gastrointest Endosc 2007; 66: $945-952$

[26] Togashi K, Nemoto D, Utano K et al. Blue laser imaging endoscopy system for the early detection and characterization of colorectal lesions: a guide for the endoscopist. Therap Adv Gastroenterol 2016; 9: $50-56$

[27] Singh R, Jayanna M, Navadgi S et al. Narrow-band imaging with dual focus magnification in differentiating colorectal neoplasia. Dig Endosc 2013; 25: (Suppl. 02): 16-20

[28] Ogiso K, Yoshida N, Siah KT et al. New-generation narrow band imaging improves visibility of polyps: a colonoscopy video evaluation study. J Gastroenterol 2016; 51: 883-890
[29] Horimatsu T, Sano Y, Tanaka S et al. Next-generation narrow band imaging system for colonic polyp detection: a prospective multicenter randomized trial. Int J Colorectal Dis 2015; 30: 947 - 954

[30] Fukuda H, Miura Y, Hayashi Y et al. Linked color imaging technology facilitates early detection of flat gastric cancers. Clin J Gastroenterol 2015; 8: 385-389

[31] Suzuki T, Hara T, Kitagawa Y et al. Linked-color imaging improves endoscopic visibility of colorectal nongranular flat lesions. Gastrointest Endosc 2017; 86: 692 -697

[32] Aminalai A, Rösch T, Aschenbeck J et al. Live image processingdoes not increase adenoma detection rate during colonoscopy: A randomized comparison between FICE and conventional imaging. Am J Gastroenterol 2010; 105: 2383-2388

[33] Hong SN, Choe WH, Lee JH et al. Prospective, randomized, back-toback trial evaluating the usefulness of i-SCAN in screening colonoscopy. Gastrointest Endosc 2012; 75: 1011-1021 

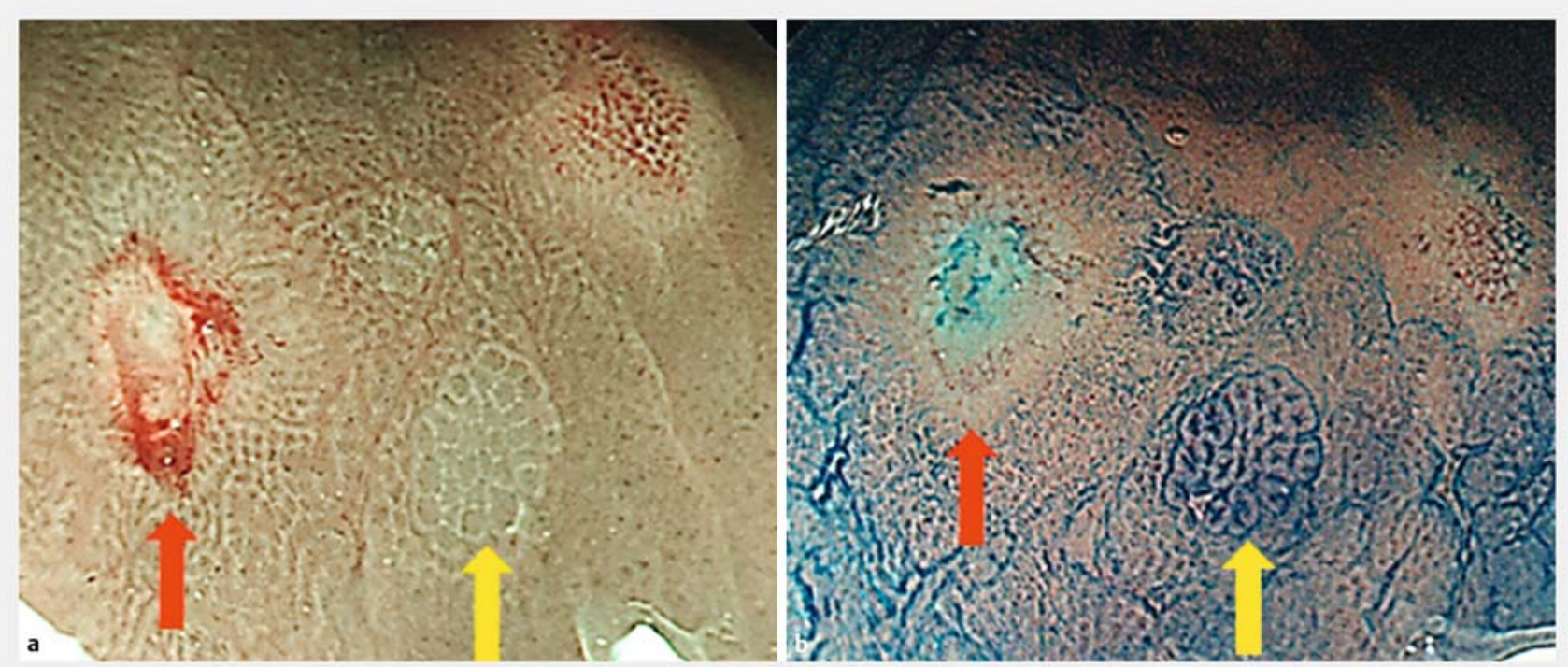

- Supplementary Fig. 1 Identification of ACF with image-enhanced endoscopy (IEE). a ACF consisting of large crypts with white pericryptal zones were observed in the lower rectal region using magnified narrow-band imaging (yellow arrow). To verify the diagnosis of ACF, the adjacent mucosa was marked by argon plasma coagulation (red arrow). b Methylene blue staining was performed on the referral area for ACF observation. Typical ACF, which consisted of large crypts densely stained with methylene blue, were detected in the corresponding location. 